\title{
SUPERINTEGRABLE POTENTIALS AND SUPERPOSITION OF HIGGS OSCILLATORS ON THE SPHERE $S^{2}$
}

\author{
MANUEL F. RAÑADA \\ Departamento de Física Teórica, Facultad de Ciencias \\ Universidad de Zaragoza, 50009 Zaragoza, Spain \\ E-mail: mfran@posta.unizar.es \\ TERESA SANZ-GIL and MARIANO SANTANDER \\ Departamento de Física Teórica, Facultad de Ciencias \\ Universidad de Valladolid, 47011 Valladolid, Spain \\ E-mail: santander@fta.uva.es
}

To Prof. W. M. Tulczyjew, with respect on the occasion of his 70th birthday

\begin{abstract}
The spherical version of the two-dimensional central harmonic oscillator, as well as the spherical Kepler (Schrödinger) potential, are superintegrable systems with quadratic constants of motion. They belong to two different spherical "Smorodinski-Winternitz" families of superintegrable potentials. A new superintegrable oscillator have been recently found in $S^{2}$. It represents the spherical version of the nonisotropic $2: 1$ oscillator and it also belongs to a spherical family of quadratic superintegrable potentials. In the first part of the article, several properties related to the integrability and superintegrability of these spherical families of potentials are studied. The second part is devoted to the analysis of the properties of the spherical (isotropic and nonisotropic) harmonic oscillators.
\end{abstract}

1. Introduction. A Hamiltonian system that is integrable (in the Liouville-Arnold sense) and possesses more constants of motion than degrees of freedom is called superintegrable (see [1], [3], [4], [6-8], [10], [12-14], [19], [21], and references therein). If the number $N$ of independent constants takes the value $N=2 n-1$ ( $n$ is the number of degrees of freedom) then the system is called maximally superintegrable. The two central

2000 Mathematics Subject Classification: Primary 37J35; Secondary 70H06, 37J15.

Key words and phrases: integrability, separability, superintegrable systems, spaces of constant curvature, quadratic constants of motion.

Support of Spanish projects, BFM-2002-03773, BFM-2000-1066 and FPA-2000-1252 (Madrid), and CO2-399 (Castilla y Leon) is acknowledged.

The paper is in final form and no version of it will be published elsewhere. 
euclidean potentials known as Bertrand systems, isotropic harmonic oscillator and Kepler problem, are two classical examples of maximal superintegrability.

Fris, Mandrosov et al. [5] studied the euclidean $n=2$ systems which admit separability in two different coordinate systems, and obtained four families $\mathcal{V}^{r}, r=a, b, c, d$, of superintegrable potentials with constants of motion linear or quadratic in the momenta. In fact, if we call a system superseparable whenever it admits Hamilton-Jacobi separation of variables (Schroedinger in the quantum case) in more than one coordinate system, then quadratic superintegrability (superintegrability with linear or quadratic constants of motion) can be considered as a property arising from superseparability. The first two families, $\mathcal{V}^{a}$ and $\mathcal{V}^{b}$, were directly related to the harmonic oscillator

$$
\begin{aligned}
V^{a} & =\frac{1}{2} \omega_{0}^{2}\left(4 x^{2}+y^{2}\right)+\frac{k_{2}}{y^{2}}+k_{3} x, \\
V^{b} & =\frac{1}{2} \omega_{0}^{2}\left(x^{2}+y^{2}\right)+\frac{k_{2}}{x^{2}}+\frac{k_{3}}{y^{2}} .
\end{aligned}
$$

The other two families, $\mathcal{V}^{c}$ and $\mathcal{V}^{d}$, were related to the Kepler problem. In fact, $V^{a}$ and $V^{b}$ can be considered as the more general deformations of the 1:1 and 2:1 oscillators $\left(k_{2}\right.$, $k_{3}$, representing the intensity of the deformation) preserving quadratic superintegrability (the three-dimensional generalizations of these potentials have been studied in [4]).

The spherical versions of the Bertrand systems (harmonic oscillator and Kepler problem) were studied by Schroedinger [18] and Higgs [9] (see also [8], [10], [11], [20]). Recently, the existence of superintegrable systems, and the properties of the Harmonic oscillators, have been analyzed on the two-dimensional sphere $S^{2}$ and on the hyperbolic plane $H^{2}$ using the curvature $\kappa$ as a parameter [15-17]. The main objective of this article is to continue with the study of these systems in the case of the sphere $S^{2}$. We will focus our study on some interesting dynamical properties closely related to the geometric properties of the sphere.

The paper is organized as follows: In Section 2 we introduce the coordinate systems on the sphere and we analyze the existence of symmetries and first integrals, as well as the separability on $S^{2}$. In Section 3 we study the spherical oscillator and the spherical Kepler problem and in Section 4 we interpret the family $\mathcal{U}^{b}$ (spherical version of $\mathcal{V}^{b}$ ) as a superposition of three 1:1 harmonic oscillators whose centers are at the vertices of a sphere's octant. In Section 5 we identify the 2:1 spherical oscillator introduced in [17] as a superposition of two Higgs oscillators whose centers are separated a quadrant $\pi / 2$ on $S^{2}$. In fact we will prove that this property can be extended to the case of the spherical $n: 1$ oscillator. In Section 6 we introduce a new superintegrable potential that is interpreted as a spherical 1/2:1 oscillator. Finally, in Section 7 we make some final comments.

\section{Geometry and dynamics on $S^{2}$}

2.1. Coordinate systems on $S^{2}$. The unit of length on $S^{2}$ will be chosen so that the sphere has curvature 1 (and hence radius $R=1$ when seen as a surface imbedded in a euclidean ambient space $E^{3}$ ). Thus any of the coordinates we will use can be equivalently seen either intrinsically, as lengths along geodesics of $S^{2}$, or extrinsically as subtended angles from the sphere center. 
When convenient we will also use a geometric, coordinate free language to facilitate the description and reference to specific superintegrable potentials on the sphere. With this aim in view we will denote by $\{N, S\},\{O, A\}$, and $\{E, W\}$, three pairs of mutually antipodal points on $S^{2}$. The geographical flavour is obvious: $N$ and $S$ are the North and South poles, and the four points $O, E, A, W$, are placed at $\pi / 2$ distances on the Equator. $O$ will be here considered as the origin point, $A$ is its antipodal, and points $E, N, W, S$, are all at distance $\pi / 2$ from $O$ in the East, North, West, and South directions respectively. Some intermediate points will be denoted by using the nautical convention: thus $O E$ is a point midway between $O$ and $E$, lying on the geodesic determined by $O$ and $E$ at a distance $\pi / 4$ from each of them; strictly speaking $O E$ refers to a pair of antipodal points, as there are two such midway points along the 'short' and 'long' geodesic arcs joining $O$ and $E$.

As far as coordinates themselves, it is better not to restrict to any single coordinate system, as superposition properties which are rather obscure in one set of coordinates may be much clearer in another one. Hence for any point $P \in S^{2}$ we will use:

1) The three (unoriented) distances $r, \widetilde{x}, \widetilde{y}$, from $P$ to the three points $O, E, N$.

2) The three (suitably oriented) distances $\widetilde{r}, x, y$, from $P$ to the three geodesics determined by the pairs $(E, N),(N, O)$ and $(O, E)$ respectively, which are the polar circles of the three points $O, E$, and $N$. These geodesics are are the two meridians at longitude $0, \pi$; the two meridians at longitude $\pi / 2,3 \pi / 2$; and the Equator.

3) The three (suitably oriented) distances $w, v, u$, intercepted on these former geodesics from the points $E, O$, and $N$, to the foot of the orthogonal geodesics from $P$. That is, if $P_{E N}$ denotes the orthogonal projection of $P$ on the geodesic $(E, N)$ then $w$ is the distance from $E$ to $P_{E N}$ that can be identified with the polar angle of $P$ at $O$. Similarly $v, u$ represent distances to the points $P_{O N}, P_{O E}$. Notice it would be more symmetrical to use the complement of $v$ instead, which is measured from $N$, but to match with the notation used in [15], [17], we will not do this.

The notation with tilde reflects the obvious complementarity relations:

$$
r+\widetilde{r}=\pi / 2, \quad y+\widetilde{y}=\pi / 2, \quad x+\widetilde{x}=\pi / 2,
$$

and we will also introduce the complements $\widetilde{w}, \widetilde{v}, \widetilde{u}$ to $w, v$ and $u$,

$$
\widetilde{w}=\pi / 2-w, \quad \widetilde{v}=\pi / 2-v, \quad \widetilde{u}=\pi / 2-u .
$$

The pair $(r, w)$ can be considered as the spherical polar coordinates of $P$ with center at $O$ and polar axis $(O, E)$. The first coordinate is the distance from $O$ to the point $P$, and the second is the angle made by $(O, E)$ with the geodesic $(O, P)$. Similarly the pairs $(\widetilde{x}, \widetilde{v})$ and $(\widetilde{y}, u)$ are also sets of polar coordinates with centers at $E$ and $N$, and polar axes $(E, N)$ and $(N, O)$, respectively.

The pair $(u, y)$ represents the (geodesic) parallel coordinates of $P$ relative to the origin $O$ and base geodesic $(O, E)$. The first coordinate $u$ is the distance measured along the base geodesic, while the second coordinate $y$ is another distance but now measured along the geodesic by $P$ orthogonal to $(O, E)$. Similarly the pairs $(w, \widetilde{r})$ and $(\widetilde{v}, x)$ are also sets of (geodesic) parallel coordinates, with base point and base geodesic $(E,(E, N))$ and $(N,(N, O))$ respectively. 
As displayed by notation, in the sphere $S^{2}$ there is no essential difference between the 'polar' and 'parallel' coordinate systems, but it is still better to keep them as two alternative systems. Standard spherical coordinates $(\theta, \phi)$, as usually defined are related to ours by $\theta=r, \phi=w$, but would be related otherwise if the polar axis were taken differently. We have preferred not to use this standard notation, as the play with three systems of coordinates is essential.

2.2. Symmetries, first integrals and separability on $S^{2}$. We will consider systems on $S^{2}$ described by natural Lagrangians $L$ of mechanical type; that is, a kinetic term $T$ determined by the Riemannian metric minus a potential $U$ depending on coordinates (if $U=0$ we have the geodesic motion on $S^{2}$ ). Let us denote by $P_{1}, P_{2}$, and $J$ the three momenta associated to the three one-parameter isometries of $S^{2}$ (corresponding to rotations around $N, E$ and $O)$. In polar $(r, w)$ coordinates they take the form

$$
\begin{aligned}
P_{1} & =(\cos w) v_{r}-(\cos r \sin r \sin w) v_{w}, \\
P_{2} & =(\sin w) v_{r}+(\cos r \sin r \cos w) v_{w}, \\
J & =\left(\sin ^{2} r\right) v_{w},
\end{aligned}
$$

and in parallel coordinates $(u, y)$ are given by

$$
\begin{aligned}
P_{1} & =\cos ^{2} y v_{u}, \\
P_{2} & =(\sin u \cos y \sin y) v_{u}+(\cos u) v_{y}, \\
J & =(\cos u \cos y \sin y) v_{u}-(\sin u) v_{y} .
\end{aligned}
$$

If the Lagrangian $L$ is endowed with an exact Noether symmetry then it possesses a constant of motion linear in these three momenta. As an example, if the potential $U$ is independent of $w$ (when written in polar $(r, w)$ coordinates) then the momentum $J$ is an integral of motion.

It is known (see e.g. [1], [2]) that, (i) a necessary condition for Hamilton-Jacobi (HJ) separability of a natural Hamiltonian $H=T+U$ in a particular coordinate system is the separabilty of the corresponding geodesic motion, and (ii) the HJ-separability implies the existence of a fundamental set of first integrals in involution quadratic or linear in the momenta. As stated above, linear constants only arise in the very particular case of exact Noether symmetries; quadratic constants are sums of a first term quadratic in the momenta and a second term $W$ depending only on coordinates.

A potential $U$ that is HJ-separable in one particular coordinate system on $S^{2}$ will depend on two arbitrary single-variable functions, $F$ and $G$. The coordinate systems separating the Laplace operator on $S^{2}$ are the elliptical ones and its non-generic degeneracies, which are the 'polar', the 'parabolic' and the 'parallel' ones when the interfocal distance is respectively $0, \pi / 2$, and $\pi$. Next we give the particular expressions for the different separable cases:

(i) A potential $U$ which is separable in a 'polar' coordinate system on $S^{2}(\operatorname{say}(r, w))$ must have the following expression:

$$
U=F(r)+\frac{G(w)}{\sin ^{2}(r)}, \quad I=J^{2}+W(r, w)
$$


where $F(r)$ and $G(w)$ are arbitrary single-variable functions. The quadratic part of the extra constant of motion is $J^{2}$ and the actual form of $W$ depends on $F$ and $G$. The particular case $G=0$ corresponds to a central potential; then the integral $I$ becomes $I=J^{2}$. The family of these integrable potentials will be denoted as $U\left[J^{2}\right]$.

(ii) A potential which is separable in a 'parallel' coordinate system, say $(u, y)$ or $(x, v)$, on $S^{2}$ should be of either form:

$$
\begin{array}{ll}
U=F(y)+\frac{G(u)}{\cos ^{2}(y)}, & I=P_{1}^{2}+W(u, y), \\
U=F(x)+\frac{G(v)}{\cos ^{2}(x)}, & I=P_{2}^{2}+W(v, x),
\end{array}
$$

where again $W$ is a function of coordinates whose form depends on $F, G$. Separable potentials of this class can be denoted by $U\left[P_{1}^{2}\right]$ and $U\left[P_{2}^{2}\right]$.

(iii) A potential which is separable in 'parabolic' coordinates $(r+y, r-y)$ or $(r+x, r-x)$ on $S^{2}$ should be of the form:

$$
\begin{array}{ll}
U=\frac{1}{2 \sin r \cos y}(F(r+y)+G(r-y)), \quad I=J P_{1}+W(r+y, r-y), \\
U=\frac{1}{2 \sin r \cos x}(F(r+x)+G(r-x)), \quad I=J P_{2}+W(r+x, r-x) .
\end{array}
$$

Separable potentials of this class can be denoted by $U\left[J P_{1}\right]$ and $U\left[J P_{2}\right]$.

Quadratically superintegrable systems are separable in at least two different coordinate systems, thus having the previous structures in at least two different forms. As an example, the family of potentials separable in both $(r, w)$ 'polar' and $(u, y)$ 'parallel' coordinate systems, will consist of all those superintegrable potentials belonging to the intersection of the sets $U\left[J^{2}\right]$ and $U\left[P_{1}^{2}\right]$.

3. The spherical Higgs oscillator, 2:1 oscillator and Kepler potentials. Let us consider the following spherical Lagrangian

$$
L=\frac{1}{2}\left(v_{r}^{2}+\sin ^{2} r v_{w}^{2}\right)-\frac{1}{2} \omega_{0}^{2} U_{11}(O), \quad U_{11}(O)=\tan ^{2} r .
$$

It represents (in polar coordinates $(r, w)$ ) the spherical version of the isotropic oscillator (Higgs oscillator [9]). It separates the sphere $S^{2}$ in two halves through an infinite potential barrier on the great circle $r=\pi / 2$, which geographically can be described as the two meridians $u=\pi / 2$ and $u=3 \pi / 2$. Classical motion is confined to each half, where the potential has a basin-like character with a minimum at $O$ and another one at its antipodal point $A$; near these points the potential is quadratic in the distance to $O$ or to $A$, thus reducing to the euclidean harmonic oscillator $r^{2}$ in the suitable tangent approximation. We shall refer to either $O$ or $A$ as the oscillator 'center'. By use of elementary relations the potential can be alternatively written in several equivalent ways in the three coordinate systems $(r, w),(u, y)$, and $(x, v)$ :

$$
U_{11}(O)=\tan ^{2} r=\tan ^{2} y+\frac{\tan ^{2} u}{\cos ^{2} y}=\tan ^{2} x+\frac{\tan ^{2} v}{\cos ^{2} x}
$$

displaying the fact that the Higgs oscillator is separable simultaneously in these three coordinate systems. In fact, it is a quadratic superintegrable potential with three inde- 
pendent constants of motion: two quadratic constants, with quadratic parts given by $P_{1}^{2}$ and $P_{2}^{2}$, and the angular momentum $J$ itself

$$
\begin{aligned}
& I_{1}=P_{1}^{2}+\omega_{0}^{2}(\tan r \cos w)^{2}, \\
& I_{2}=P_{2}^{2}+\omega_{0}^{2}(\tan r \sin w)^{2}, \\
& I_{3}=J .
\end{aligned}
$$

The two quadratic functions, $I_{1}$ and $I_{2}$, together with the square of $J$, add up to the total energy, that is, $I_{1}+I_{2}+J^{2}=H$. The Higgs oscillator is a central potential, and on each geodesic through $O$ it reduces to the one-dimensional harmonic oscillator $\tan ^{2} r$, thus entitling the denomination 1:1 or isotropic oscillator appearing, together with its center, in the notation $U_{11}(O)$.

The Higgs potential $U_{11}(O)$ can also be expressed in parallel $(u, y)$ coordinates

$$
U_{11}(O)=\tan ^{2} y+\frac{\tan ^{2} u}{\cos ^{2} y}
$$

then the two quadratic integrals, $I_{1}$ and $I_{2}$, become

$$
I_{1}=P_{1}^{2}+\omega_{0}^{2} \tan ^{2} u, \quad I_{2}=P_{2}^{2}+\omega_{0}^{2}\left(\frac{\tan y}{\cos u}\right)^{2} .
$$

In parallel $(u, y)$ coordinates the potential of the spherical 2:1 harmonic oscillator is given by

$$
U_{21}(O ; N):=\tan ^{2} y+\frac{\tan ^{2}(2 u)}{\cos ^{2} y} .
$$

It is a non-central but superintegrable potential with

$$
\begin{aligned}
& I_{1}=P_{1}^{2}+\omega_{0}^{2} \tan ^{2}(2 u), \\
& I_{2}=P_{2}^{2}+J^{2}+\omega_{0}^{2}\left(\frac{\tan y}{\cos (2 u)}\right)^{2}, \\
& I_{3}=J P_{2}+\omega_{0}^{2}(\sin u \cos u)\left(\frac{\tan y}{\cos (2 u)}\right)^{2},
\end{aligned}
$$

as constants of motion. In this case $I_{1}$ and $I_{2}$ are directly related to the Hamiltonian by the equation $I_{1}+I_{2}=H$.

In the euclidean limit around $O($ small $u, y)$ this potential reduces to $4 u^{2}+y^{2}$, and hence is an anisotropic euclidean 2:1 oscillator. Along the geodesic $O N(u=0)$ through $O$, it reduces to $\tan ^{2}(y)$, which approximates to $y^{2}$ near $O$. And along the orthogonal geodesic $O E(y=0)$, it reduces to $\tan ^{2}(2 u)$, which near $O$ approximates to $4 u^{2}$. As both $u, y$ are distances along these geodesics, this potential can be considered as an anisotropic 2:1 oscillator; it is not a central potential, and grows from a $4 u^{2}+y^{2}$ approximate behaviour near $O$ to an infinite value along the boundary of the wedge determined by the two meridians $u=\pi / 4$ and $u=-\pi / 4$. It should be noted that this infinite value is reached at a distance $\pi / 2$ from $O$ along the $O N$ direction, but at half this distance (i.e., $\pi / 4$ ) along the $O E$ direction. The two meridians determining the basin of the point $O$ are indeed parts of two great circles both through $N$ and orthogonal, dividing the sphere into four 'wedges'. The point $O$ is at the center of one wedge, and of course the points $E, A, W$, 
could as well be considered as the potential centers in their corresponding wedge; all the four wedges are equivalent because of the periodicity of the potential. To describe unambiguously such a 2:1 potential we should specify one of its centers (in our example $O$ ) and next should distiguish the directions from $O$ corresponding to the ' 2 ' and to the ' 1 ' growing rate; as these directions are orthogonal, it will be enough to simply specify one of them, and conventionally we will choose to indicate the 1 direction along which the infinite value for the potential is reached after a distance $\pi / 2$ (in our example $O N$ ); only the point $N$ will be appended to the notation, as the origin has been already registered. This explains the symbol $U_{21}(O ; N)$. This potential is separable in both parallel $(u, y)$ coordinates and in 'parabolic' coordinates $(r+x, r-x)$, and can be presented by the two alternative expressions

$$
U_{21}(O ; N)=\tan ^{2} y+\frac{\tan ^{2}(2 u)}{\cos ^{2} y}=\frac{1}{2 \sin r \cos x}(F(r+x)+G(r-x))
$$

with appropriate expressions of $F$ and $G$.

A potential as $U_{21}(O ; E)$ would have the same origin but the ' 1 ' and ' 2 ' directions interchanged. It is in the same class as the former one, in the sense that a certain sphere isometry would map one into another, but as a matter of fact it is actually a different potential having the euclidean limit $x^{2}+4 v^{2}$ around $O$ :

$$
U_{21}(O ; E):=\tan ^{2} x+\frac{\tan ^{2}(2 v)}{\cos ^{2} x} .
$$

The spherical Kepler potential $[8,9,15,18]$ is another remarkable central potential on $S^{2}$ :

$$
U_{K}(O):=-\frac{k}{\tan r} .
$$

Its value at the origin $O$ equals $-\infty$, growing to a zero value on the great circle $r=\pi / 2$ and then to a positive infinite value at the antipodal point $A$. This will be referred to as the Kepler potential with center at $O$, while again it has clearly two antipodal centers. Unlike for the oscillator these two centers display here a sign difference, and coincide with the points where the potential is infinite. The spherical Kepler potential is separable in three coordinate systems: polar $(r, w)$, and two parabolic systems $(r+x, r-x)$ or $(r+y, r-y)$, with alternative expressions:

$$
\begin{aligned}
U_{K}(O)=\frac{-k}{\tan r} & =\frac{-k}{2 \sin r \cos x}(\cos (r+x)+\cos (r-x)) \\
& =\frac{-k}{2 \sin r \cos y}(\cos (r+y)+\cos (r-y))
\end{aligned}
$$

It has three constants of motion in addition to the energy: the angular momentum $J$ and two quadratic integrals with quadratic part of the form $J P_{1}$ and $J P_{2}$

$$
\begin{aligned}
& I_{1}=J P_{1}-k \sin w, \\
& I_{2}=J P_{2}+k \cos w, \\
& I_{3}=J .
\end{aligned}
$$


Likewise the spherical Kepler potentials centered at the points $E$ or $N$ would be:

$$
U_{K}(E):=-\frac{k}{\tan \widetilde{x}}, \quad U_{K}(N):=-\frac{k}{\tan \widetilde{y}},
$$

whose euclidean limits around $O$ turn out to be the linear euclidean potentials $-k x,-k y$.

4. The spherical 'harmonic oscillator' superintegrable family. Let us consider the family of superintegrable potentials on $S^{2}$ associated to the Higgs oscillator. This family is characterized by the existence of three constants of motion $I_{r}, r=1,2,3$, with $J^{2}, P_{1}^{2}$ and $P_{2}^{2}$ as quadratic parts. These three functions are functionally independent but their sum is just the Hamiltonian

$$
I_{1}+I_{2}+I_{3}=H
$$

which is always a constant of motion. Thus this family could be denoted as $\mathcal{U}\left[J^{2}, P_{1}^{2}, P_{2}^{2}\right]$ making reference to this fact; this same family will also be called $\mathcal{U}^{b}$ to match the notations in [15]. The more general potential in $\mathcal{U}^{b}$ turns out to be a 'linear superposition' $k_{1} U_{1}^{b}+$ $k_{2} U_{2}^{b}+k_{3} U_{3}^{b}$ of the following three fundamental potentials:

$$
U_{1}^{b}:=\tan ^{2} r, \quad U_{2}^{b}:=\frac{1}{\sin ^{2} x}, \quad U_{3}^{b}:=\frac{1}{\sin ^{2} y},
$$

with arbitrary real constants $k_{1}, k_{2}, k_{3}$. The euclidean limit in the neigbourhood of $O$ corresponds to taking $r, x, y \ll 1$, carrying directly to the Euclidean family $\mathcal{V}^{b}$ :

$$
\mathcal{V}^{b} \equiv k_{1} r^{2}+k_{2} \frac{1}{x^{2}}+k_{3} \frac{1}{y^{2}}
$$

The potential $U_{1}^{b}$ represents a Higgs oscillator centered at the origin $O$. The other two potentials, $U_{2}^{b}$ and $U_{3}^{b}$, can be interpreted as two 'one-dimensional' spherical centrifugallike barriers 'centered' at the two orthogonal great circles, $x=0$ and $y=0$, through $O$. If we express these two potentials, $U_{2}^{b}$ and $U_{3}^{b}$, in terms of the complements $\widetilde{x}, \widetilde{y}$, we obtain

$$
U_{2}^{b}=\frac{1}{\sin ^{2} x}=\frac{1}{\cos ^{2} \widetilde{x}}=\tan ^{2} \widetilde{x}+1
$$

and a similar expression for $U_{3}^{b}$. Thus, the generic potential $U^{b}$ in the family $\mathcal{U}^{b}$ can be rewritten as follows

$$
U^{b}=k_{1} \tan ^{2} r+k_{2} \tan ^{2} \widetilde{x}+k_{3} \tan ^{2} \widetilde{y}+\left(k_{2}+k_{3}\right)
$$

where the constant term $k_{2}+k_{3}$ can be disregarded. As $\widetilde{x}, \widetilde{y}$ are the distances to the two points $E, N$, under this form the three terms are immediately identified as three spherical harmonic oscillators, with centers at the three vertices $O, E$, and $N$, of a sphere's octant.

Hence an alternative way of representing the family $\mathcal{U}^{b}$ is

$$
\mathcal{U}^{b} \equiv \mathcal{U}\left[J^{2}, P_{1}^{2}, P_{2}^{2}\right] \equiv\left\langle U_{11}(O), U_{11}(E), U_{11}(N)\right\rangle
$$

where $\langle\cdots\rangle$ denotes the linear span of the functions enclosed. Each oscillator provides an infinite barrier at the polar circle of its origin. In the generic case where the three constants $k_{1}, k_{2}, k_{3}$, are different from zero, this potential divides the sphere into eight 'octants' separated by infinite barriers. The configuration of centers has the symmetry group of a cube and there are transformations (the rotation around the octant center by an angle of $2 \pi / 3)$ which permutes cyclically the three Higgs oscillators $U_{11}(O), U_{11}(E)$, 
and $U_{11}(N)$; each of these Higgs oscillators has two antipodal centers and the centers of any two different oscillators are separated by $\pi / 2$. In the form (28), the euclidean limit around $O$ requires a suitable approximation to both functions $\tan ^{2} \widetilde{x}, \tan ^{2} \widetilde{y}$ (near $O$ both $\widetilde{x}, \widetilde{y}$ must be close to $\pi / 2$ ). While the limit is again (26), strictly speaking the possibility of interpreting the centrifugal barriers as oscillators is lost in the euclidean limit.

\section{Superposition of isotropic spherical oscillators and anisotropic $n: 1$ spher-}

ical oscillators. Now let us consider the potential $U$ obtained as a superposition of two 1:1 oscillators with centers separated by a distance $\pi / 2$; to be specific, let us consider two Higgs oscillators centered at points $O$ and $E$ so that, as was proved in Sec. 4, the superposition is again a member of the family $\mathcal{U}^{b}$. Making use of the $(u, y)$ system of coordinates, we have

$$
U_{11}(O):=\tan ^{2} y+\frac{\tan ^{2} u}{\cos ^{2} y}, \quad U_{11}(E):=\tan ^{2} y+\frac{\tan ^{2}(u-\pi / 2)}{\cos ^{2} y} .
$$

The sum of two such potentials is:

$$
U_{11}(O)+U_{11}(E)=2 \tan ^{2} y+\frac{\tan ^{2} u+\tan ^{2}(u-\pi / 2)}{\cos ^{2} y}
$$

where

$$
\tan ^{2} u+\tan ^{2}(u-\pi / 2)=4 \tan ^{2}(2(u-\pi / 4))+2
$$

and so (31) becomes

$$
\begin{aligned}
U_{11}(O)+U_{11}(E) & =2 \tan ^{2} y+\frac{4 \tan ^{2}(2(u-\pi / 4))+2}{\cos ^{2} y} \\
& =4\left(\tan ^{2} y+\frac{\tan ^{2}(2(u-\pi / 4))}{\cos ^{2} y}\right)+2 .
\end{aligned}
$$

The term inside the large parentheses is immediately recognized as a 2:1 spherical oscillator centered at $(u=\pi / 4, y=0)$ corresponding to the point $O E$ placed midway between the centers $O$ and $E$ of the two original $U_{11}$ oscillators, and with '1' direction towards the point $N$. Thus we have found:

$$
U_{11}(O)+U_{11}(E)=4 U_{21}(O E ; N)+2
$$

reproducing exactly the coefficients in the identity (32) even though this equation refers to a $2 \mathrm{D}$ potential where the terms depending on $y$ arrange automatically due to the specific 'separable in $(u, y)$ structure'.

Conversely, the nonisotropic 2:1 harmonic oscillator can be expressed as a linear superposition of two isotropic oscillators plus constant terms. For the 2:1 oscillator $U_{21}(O ; N)$ centered at $O$ the decomposition is:

$$
U_{21}(O ; N)=\frac{1}{4}\left(U_{11}(O E)+U_{11}(O W)-2\right) .
$$

The infinite barriers in $U_{21}(O ; N)$ are simply the union of the infinite barriers in the two component 1:1 oscillators, which are two orthogonal great circles, thus separating the sphere into four identical wedges as is known to be the case for $U_{21}(O ; N)$. 
Once this fact has been recognized, the extension of this property to the case of any number of component oscillators is immediate. Let us consider 3 isotropic $U_{11}$ harmonic oscillators with centers on the line $y=0$ and spaced $\pi / 3$ apart. To be specific, let us place the centers at the points $P_{1}=(-\pi / 3,0), O=(0,0)$, and $P_{3}=(\pi / 3,0)$. The identity analogous to $(32)$ is

$$
\tan ^{2}(u+\pi / 3)+\tan ^{2}(u)+\tan ^{2}(u-\pi / 3)=9 \tan ^{2}(3 u)+6
$$

and hence for the sum of the three 1:1 oscillators centered at these points we get:

$$
\begin{aligned}
U_{11}\left(P_{1}\right)+U_{11}(O)+U_{11}\left(P_{3}\right) & =3 \tan ^{2} y+\frac{9 \tan ^{2}(3 u)+6}{\cos ^{2} y} \\
& =9\left(\tan ^{2} y+\frac{\tan ^{2}(3 u)}{\cos ^{2} y}\right)+6 .
\end{aligned}
$$

This suggests considering the potential:

$$
U_{31}(O ; N):=\tan ^{2} y+\frac{\tan ^{2}(3 u)}{\cos ^{2} y}
$$

reducing in the euclidean limit around $O$ to $9 u^{2}+y^{2}$, as a good candidate for an anisotropic $3: 1$ spherical oscillator, with center at $O$ and ' 1 ' direction towards $N$. For this potential, the sphere is divided into 6 identical wedges, on whose boundaries the potential becomes infinite. The boundary of the wedge centered at $O$ are the two meridians $u=-\pi / 6$ and $u=\pi / 6$, and again the potential grows in the ' 3 ' direction faster than in the ' 1 ' direction, reaching an infinite value at a distance $\pi / 6$ from $O$, that is, one third of the distance required to reach infinite value along the ' 1 ' direction. The same situation repeats inside each wedge and this potential has three pairs of antipodal centers; these centers of the $3: 1$ oscillator coincide with the centers of any of the oscillator components, unlike the 2:1 case, and this difference depends on the different parity of the number of oscillator components.

The fact is general for any value of $n=2,3, \ldots$ Consider $n$ pairs of antipodal points placed on a great circle (say $y=0$ ) so that they are equispaced (hence next neighbours are spaced $\pi / n$ apart), and let $u_{i}, i=1, \ldots, n$, be the $u$ coordinates of one point in each pair. Let us choose the set of $u_{i}$ given by $u= \pm(\pi /(2 n)+m \pi / n)$ with $m=1, \ldots, n / 2$, when $n$ is even, and by $u=0, u= \pm m \pi / n$, with $m=1, \ldots,(n-1) / 2$, when $n$ is odd. In either case we have the identity:

$$
\sum_{i}^{n} \tan ^{2}\left(u-u_{i}\right)=n^{2} \tan ^{2}(n u)+n(n-1) .
$$

which for the sum of the corresponding $U_{11}$ oscillator potentials centered at the points $\left(u=u_{i}, y=0\right)$ gives

$$
n \tan ^{2} y+\frac{n^{2} \tan ^{2}(n u)+n(n-1)}{\cos ^{2} y}=n^{2}\left(\tan ^{2} y+\frac{\tan ^{2}(n u)}{\cos ^{2} y}\right)+n(n-1) .
$$

Again the potential

$$
U_{n 1}(O ; N):=\tan ^{2} y+\frac{\tan ^{2}(n u)}{\cos ^{2} y}
$$


might be considered as a spherical $n: 1$ oscillator. The infinite barriers in this potential separate the sphere into $2 n$ wedges, all crossing at $N$, and inside each wedge there is a center, $O$ being always one of them. Around $O$ the potential behaves like $n^{2} u^{2}+y^{2}$, but it grows to infinity at the wedge boundaries.

The structure of the potentials $U_{n 1}(O ; N)$ displays directly the existence of a constant of motion quadratic in the momenta, beside the energy. It would be interesting to search further constants for these potentials. It seems that these $n: 1$ oscillator are not quadratically superintegrable unless $n=2$, but we do not know whether these potentials have other constants of higher order in the momenta.

6. The 1/2:1 spherical oscillators. By different groupings in the previous sums, a $4: 1$ oscillator (potential $U_{41}$ ) can be considered in two different ways: as the sum of four isotropic $U_{11}$ oscillators and also as a sum of two 2:1 oscillators. This corresponds to different groupings of the sums, and may allow a wealth of different possibilities for decompositions in the general $n: 1$ case. One can thus wonder whether this chain of $n: 1$ spherical harmonic oscillators starting out of the 1:1 case can be produced backwards, allowing the 1:1 oscillator to be expressed as a superposition of two other relevant potentials.

We now start with the following trigonometric identity:

$$
\tan ^{2}\left(\frac{u-u_{0}}{2}\right)+\tan ^{2}\left(\frac{u+u_{0}}{2}\right)=4 \tan ^{2} u+2, \quad u_{0}=\frac{\pi}{2},
$$

so we have

$$
\begin{aligned}
4\left[\tan ^{2} y+\frac{\tan ^{2} u}{\cos ^{2} y}\right] & =4 \tan ^{2} y+\frac{1}{\cos ^{2} y}\left[\tan ^{2}\left(\frac{u-u_{0}}{2}\right)+\tan ^{2}\left(\frac{u+u_{0}}{2}\right)-2\right] \\
& =2 \tan ^{2} y+\frac{1}{\cos ^{2} y}\left[\tan ^{2}\left(\frac{u-u_{0}}{2}\right)+\tan ^{2}\left(\frac{u+u_{0}}{2}\right)\right]-2 .
\end{aligned}
$$

Thus, if we denote by $U_{1 / 2,1}(E ; N)$ and $U_{1 / 2,1}(W ; N)$ the following two potentials:

$$
\begin{aligned}
U_{1 / 2,1}(E ; N) & :=\tan ^{2} y+\frac{\tan ^{2}\left(\left(u-u_{0}\right) / 2\right)}{\cos ^{2} y}, \\
U_{1 / 2,1}(W ; N) & :=\tan ^{2} y+\frac{\tan ^{2}\left(\left(u+u_{0}\right) / 2\right)}{\cos ^{2} y},
\end{aligned}
$$

then we obtain

$$
U_{1 / 2,1}(E ; N)+U_{1 / 2,1}(W ; N)=4 U_{11}(O)+2 .
$$

These two new potentials are worth the name of $1 / 2: 1$ spherical oscillators. They are respectively centered at the two points $E$ (resp. $W$ ), and starting from these points along the ' 1 ' direction towards $N$ they reach an infinite value after a $\pi / 2$ distance, while along the ' $1 / 2$ ' orthogonal direction pointing to $O$ or to $A$ (resp. to $A$ or to $O$ ) they reach an infinite value only after a $\pi$ distance, that is, only at the antipodal point $W$ (resp. $E)$ of the center. The infinite barriers of each of these potentials correspond to a single meridian, that is, to a half of a complete great circle, $(N, W, S)$ with $u=3 \pi / 2$ (resp. $(N, E, S)$ with $u=\pi / 2)$.

The sum of the two $1 / 2: 1$ oscillators centered at the points $W$ and $E$, represented by the two functions $U_{1 / 2,1}$ in (44), is an isotropic 1:1 oscillators centered at the origin $O$; 
this is again the midpoint between the centers of the two superposed oscillators in their common ' $1 / 2$ ' direction.

The potential of the spherical $1 / 2: 1$ oscillator with center at the origin $O$ and ' $1 / 2$ ' direction towards $N$ is given by

$$
U_{1 / 2,1}(O ; N):=\tan ^{2} y+\frac{\tan ^{2}(u / 2)}{\cos ^{2} y} .
$$

This potential is clearly separable in the $(u, y)$ ('parallel') coordinate system, and so it belongs to the family $U\left[P_{1}^{2}\right]$. In fact the Hamiltonian $H$ can be written as $H=I_{1}+I_{2}$ with $I_{1}, I_{2}$ the following two integrals of motion:

$$
I_{1}=P_{1}^{2}+\omega_{0}^{2} \tan ^{2}(u / 2), \quad I_{2}=P_{2}^{2}+J^{2}+\omega_{0}^{2}\left(\frac{\tan y}{\cos (u / 2)}\right)^{2} .
$$

Moreover, it is superintegrable as well, since it is separable in 'parabolic' coordinates and it also belongs to the family $U\left[J P_{1}\right]$. Thus the $1 / 2: 1$ oscillator potentials are interesting for two reasons: first because they are superintegrable, and second because they may be considered as a sort of 'basic' spherical oscillators, as any other spherical oscillator either isotropic $1: 1$ or anisotropic $\mathrm{n}: 1, n=2,3, \ldots$, turns out to be ultimately a suitable linear superposition of $1 / 2: 1$ spherical harmonic oscillators.

7. Final comments and outlook. We have started with a discussion of the curvilinear systems of coordinates on the two-dimensional sphere $S^{2}$, and then we have studied the properties of some spherical superintegrable systems. Concerning the case of the harmonic oscillators on the sphere $S^{2}$, we have proved that:

(i) The nonisotropic oscillator $U_{21}$ can be considered as a superposition of two isotropic Higgs oscillators $U_{11}$ with centers separated by a distance of $\pi / 2$ on $S^{2}$.

(ii) The potential obtained as an appropriate linear superposition of $n$ isotropic Higgs oscillators can be interpreted as an $n: 1$ nonisotropic $U_{n 1}$ oscillator.

(iii) The Higgs oscillator $U_{11}$ can be considered itself as a superposition of two 1/2:1 nonisotropic $U_{1 / 2,1}$ oscillators with antipodal centers (at a distance of $\pi$ on $S^{2}$ ).

These three properties are very remarkable. First because they are dynamical characteristics that must be considered as arising from the noneuclidean geometry of the configuration space. Second, because they introduce a relation, just by linear superposition, between superintegrable potentials belonging to different families of spherical superintegrable systems. In fact, the central Higgs oscillator $U_{11}$ belongs to the family $\mathcal{U}\left[J^{2}, P_{1}^{2}, P_{2}^{2}\right]$, and the nonisotropic oscillators $U_{21}$ and $U_{1 / 2,1}$, to the families $\mathcal{U}\left[P_{1}^{2}, J P_{2}\right]$ and $\mathcal{U}\left[P_{1}^{2}, J P_{1}\right]$. Of course $U_{21}$ and $U_{1 / 2,1}$ represent the same system in the Euclidean plane, but this is not the case in the sphere.

All these characteristics underline the distinctions between the euclidean and the spherical dynamics. Because of this, it will be very convenient to study, once again, all these properties but using the curvature $\kappa$ as parameter (this formalism has already been used in $[16,17])$. It seems probable that many of the expressions obtained in Secs. 4, 5, and 6 will be $\kappa$-dependent. In any case, the above three points also introduce relations between the different families that deserve to be studied. Another point is the superinte- 
grability of the potentials $U_{n 1}$ with $n>2$. We think that these are open questions that must be investigated.

\section{References}

[1] S. Benenti, C. Chanu, and G. Rastelli, The super-separability of the three-body inversesquare Calogero system, J. Math. Phys. 41 (2000), 4654-4678.

[2] S. Benenti, C. Chanu, and G. Rastelli, Variable separation for natural Hamiltonian with scalar and vector potentials in Riemannian manifolds, J. Math. Phys. 42 (2001), 20652091.

[3] C. Daskaloyannis, Quadratic Poisson algebras of two-dimensional classical superintegrable systems and quadratic associative algebras of quantum superintegrable systems, J. Math. Phys. 42 (2001), 1100-1119.

[4] N. W. Evans, Super-integrability in classical mechanics, Phys. Rev. A 41 (1990), 5666-76.

[5] T. I. Fris, V. Mandrosov, Y. A. Smorodinsky, M. Uhlir, and P. Winternitz, On higher symmetries in quantum mechanics, Phys. Lett. 16 (1965), 354-356.

[6] C. Gonera, On the super-integrability of Calogero-Moser-Sutherland model, J. Phys. A 31 (1998), 4465-4472.

[7] C. Grosche, G. S. Pogosyan, and A. N. Sissakian, Path integral discussion for SmorodinskyWinternitz potentials I, Fortschr. Phys. 43 (1995), 453-521.

[8] C. Grosche, G. S. Pogosyan, and A. N. Sissakian, Path integral discussion for SmorodinskyWinternitz potentials II, Fortschr. Phys. 43 (1995), 523-563.

[9] P. W. Higgs, Dynamical symmetries in a spherical geometry I, J. Phys. A 12 (1979), 309-323.

[10] E. G. Kalnins, J. M. Kress, G. S. Pogosyan, and W. Miller, Completeness of superintegrability in two-dimensional constant-curvature spaces, J. Phys. A 34 (2001), 4705-4720.

[11] H. I. Leemon, Dynamical symmetries in a spherical geometry II, J. Phys. A 12 (1979), 489-501.

[12] E. McSween and P. Winternitz, Integrable and superintegrable Hamiltonian systems in magnetic fields, J. Math. Phys. 41 (2000), 2957-2967.

[13] M. F. Rañada, Superintegrable $n=2$ systems, quadratic constants of motion, and potentials of Drach, J. Math. Phys. 38 (1997), 4165-4178.

[14] M. F. Rañada, Super-integrability of the Calogero-Moser system: constants of motion, master symmetries, and time-dependent symmetries, J. Math. Phys. 40 (1999), 236-247.

[15] M. F. Rañada and M. Santander, Super-integrable systems on the two-dimensional sphere $S^{2}$ and the hyperbolic plane $H^{2}$, J. Math. Phys. 40 (1999), 5026-5057.

[16] M. F. Rañada and M. Santander, On some properties of the harmonic oscillator on spaces of constant curvature, Rep. Math. Phys. 49 (2002), 335-343.

[17] M. F. Rañada and M. Santander, On harmonic oscillators on the two-dimensional sphere $S^{2}$ and the hyperbolic plane $H^{2}$, J. Math. Phys. 43 (2002), 431-451.

[18] E. Schroedinger, A method of determining quantum mechanical eigenvalues and eigenfunctions, Proc. R.I.A. A XLVI (1940), 9-16.

[19] M. B. Sheftel, P. Tempesta, and P. Winternitz, Superintegrable systems in quantum mechanics and classical Lie theory, J. Math. Phys. 42 (2001), 659-673.

[20] J. J. Sławianowski, Bertrand systems on spaces of constant sectional curvature, Rep. Math. Phys. 46 (2000), 429-460.

[21] A. V. Tsiganov, The Drach super-integrable systems, J. Phys. A 33 (2000), 7407-7422. 